\title{
Translation of MMTV Gag requires nuclear events involving splicing motifs in addition to the viral Rem protein and RmRE
}

\author{
Ioana Boeras, Michael Sakalian and John T West
}

\begin{abstract}
Background: Retroviral Gag proteins are encoded in introns and, because of this localization, they are subject to the default pathways of pre-mRNA splicing. Retroviruses regulate splicing and translation through a variety of intertwined mechanisms, including 5'- post-transcriptional control elements, 3'- constitutive transport elements, and viral protein RNA interactions that couple unspliced and singly spliced mRNAs to transport machinery. Sequences within the gag gene termed inhibitory or instability sequences also appear to affect viral mRNA stability and translation, and the action of these sequences can be countered by silent mutation or the presence of RNA interaction proteins like HIV-1 Rev. Here, we explored the requirements for mouse mammary tumor virus (MMTV) Gag expression using a combination of in vivo and in vitro expression systems.

Results: We show that MMTV gag alleles are inhibited for translation despite possessing a functional open reading frame (ORF). The block to expression was post-transcriptional and targeted the mRNA but was not a function of mRNA transport or stability. Using bicistronic reporters, we show that inhibition of gag expression imparted a block to both cap-dependent and cap-independent translation onto the mRNA. Direct introduction of in vitro synthesized gag mRNA resulted in translation, implying a nuclear role in inhibition of expression. The inhibition of expression was overcome by intact proviral expression or by flanking gag with splice sites combined with a functional Rem-Rem response element (RmRE) interaction.

Conclusions: Expression of MMTV Gag requires nuclear interactions involving the viral Rem protein, its cognate binding target the RmRE, and surprisingly, both a splice donor and acceptor sequence to achieve appropriate signals for translation of the mRNA in the cytoplasm.
\end{abstract}

Keywords: Betaretrovirus, gag, Rem, post-transcriptional regulation

\section{Background}

Eukaryotic mRNAs typically contain intronic sequences that must be removed by ubiquitously acting splicing mechanisms prior to nuclear export. Splicing occurs cotranscriptionally and affects pre-mRNA stability, 5 ' and 3 ' end formation, nuclear export, cytoplasmic trafficking and stability, as well as translation $[1,2]$. As the premRNA emerges from PolII it is complexed with a very dynamic series of proteins that forms the mRNP (messenger ribonucleoprotein). Components of the mRNP

\footnotetext{
* Correspondence: john-west@ouhsc.edu

Department of Microbiology and Immunology, University of Oklahoma Health Sciences Center, Oklahoma City, OK, USA
}

and changes to those components regulate nuclear and cytoplasmic steps in mRNA metabolism [1-4].

Post-integration, retroviruses largely utilize host transcription and translation mechanisms to proceed through the replication cycle. Retroviruses initiate a complex transcription profile that is driven by alternative splicing and results in three distinct RNA species: unspliced genome-length transcripts, singly spliced transcripts, and fully spliced transcripts. For all retroviruses, the primary structural protein Gag is encoded by an intron in the unspliced genomic RNA. Thus, Gag translation requires unique mechanisms to overcome the default eukaryotic splicing pathway and transport an intron-containing mRNP to the cytoplasm.
C Biomed Central

() 2012 Boeras et al; licensee BioMed Central Ltd. This is an Open Access article distributed under the terms of the Creative Commons Attribution License (http://creativecommons.org/licenses/by/2.0), which permits unrestricted use, distribution, and reproduction in any medium, provided the original work is properly cited. 
In parallel with the evolving complexity of their expression programs, retroviruses have acquired increasingly complex mechanisms to regulate mRNA processing and export. One of these strategies is the adoption of suboptimal splice sites and splicing enhancers and suppressors that regulate the capacity of cellular machinery to cleave the mRNA prior to export [5-8]. This strategy is adopted by the avian sarcoma leukosis viruses (ASLV), murine leukemia virus (MLV), and other simple retroviruses, but it is also maintained in more complex retroviral species. In addition to suboptimal splicing, some betaretroviruses contain intronic RNA structures, called constitutive transport elements (CTE), which directly bridge the interaction of singlyspliced and genomic mRNAs with proteins of the TAP transport pathway [9]. Complex retroviruses add an additional level of control by encoding a regulator of RNA export from a spliced viral mRNA and utilizing a different export pathway. These proteins, the prototype of which is human immunodeficiency virus type 1 (HIV-1) Rev, interact with an RNA structural element in underspliced gag or env mRNAs and couple them to the Crm1 export pathway [10-15].

MMTV has been extensively studied as a model for breast cancer. Much emphasis has been given to defining the hormonal regulation of transcription and the mechanisms of cellular transformation [16,17]. MMTV encodes a regulatory protein Rem $[13,18]$ that is functionally equivalent to HIV Rev. Rem interacts with unspliced or singly-spliced RNA through a secondary structure, the Rem response element (RmRE), that spans the 3' end of the env gene and part of the 3'long terminal repeat (LTR) [14,19]. This interaction is required for transport of genome-length RNA from the nucleus to the cytoplasm through Crm1 $[13,18]$.

Although initially identified due to their role in transport of unspliced viral RNA from the nucleus to the cytoplasm, regulatory proteins like Rev have been shown to possess other functions. Both HIV-1 Rev and Jaagsiekte sheep retrovirus (JSRV) Rej enhance gag translation $[15,20]$. HIV-1 Rev accomplishes this by increasing the stability of gag-containing mRNA in the cytoplasm [21] and by enhancing the association of RRE-containing mRNAs with polysomes [20,22]. The mechanism of translational enhancement by JSRV Rej has not been thoroughly characterized, but is clearly independent of mRNA export [15]. The stability of HIV-1 unspliced mRNAs is regulated by inhibitory or instability sequences (INS) present in introns, including the gag ORF. These elements function in the absence of other viral genes or proteins and render the RNA unstable in the cytoplasm such that the presence of INS can result in reduced translation [21]. The RNA instability can be overcome by silent mutagenesis of the INS or by addition of Rev in trans [23]. Similarly, Butsch et al. have demonstrated that, despite efficient RNA export to the cytoplasm, gag-containing constructs fail to translate unless 5'-UTR elements, termed post-transcriptional control elements (PCE) interact with RNA helicase A [24-27]. These data further support the existence of inhibitory sequences within the gag ORF.

Relatively little is known about the basic virus-host interactions leading to MMTV expression, assembly and replication in contrast to the current understanding of MLV-, or HIV-1-host interactions. This lack of knowledge fundamentally results from an inability to bacterially propagate MMTV proviral genomes or subviral constructs containing intact gag genes derived from exogenous virus but not those from germline-encoded elements. The gag genes from endogenous murine viruses propagate in plasmid vectors; therefore, the two available MMTV infectious molecular clones pHyb-Mtv [28] and pGR102 [29] contain the gag gene from germlineencoded Mtv1 and $M t v 8$, respectively. They differ in the remainder of the viral genome. The sequence that impedes bacterial propagation of MMTV is localized in the pp21 domain of gag and has been termed the "bacterial poison sequence" [30]. An exogenous, GR strain gag gene, containing a 57-nucleotide deletion in the pp21 domain, efficiently propagated in bacteria, suggesting that the deleted region encompassed the bacterial poison sequence. Zabransky et al. silently mutated all the possible nucleotides in the sequence targeted by the deletion creating a Gag-only expression construct SM (silently mutated) that readily propagated as a bacterial plasmid and expressed Gag in eukaryotic cells [31].

However, SM Gag failed to assemble into virus-like particles or into detectable subviral structures in vitro, or in cells (unpublished data). Since the gag gene in the pHyb-Mtv infectious molecular clone (Mtv-1) makes infectious progeny upon SM Gag expression, it is clear that this gag allele is expression and assembly competent. Nevertheless, when Mtv-1 gag was introduced into the same expression construct used to successfully express SM Gag, no protein was expressed. Similar results were obtained from a highly homologous, putative human-origin, MMTV gag, HBRV (human betaretrovirus) in the same expression system. In contrast, all three gag genes expressed equivalently in cell-free, coupled, transcription-translation reactions.

We show that transcript abundance, nuclear-cytoplasmic transport, and transcript stability were equivalent among the inhibited Mtv-1 and HBRV alleles as well as Gag-producing SM allele. Our results suggest that inhibition of gag expression is a nuclear event that manifests its phenotype in the cytoplasm through translational inhibition. Interestingly, rescue of the phenotype requires the presence of the RmRE, and for gag to be in 
an intron flanked by splice donor and acceptor sites. Therefore, the history of the mRNA in the nucleus imparts an inhibitory phenotype that can be overcome by Rem and splicing motifs. Our results suggest contributions of splicing motifs and machinery and RNA export signals in the 'licensing' of the MMTV gag-containing mRNAs for translation.

\section{Results}

The silently-mutated gag expresses protein from a heterologous promoter, but wild-type Gag expression is undetectable

Three gag alleles were cloned into a cytomegalovirus (CMV) immediate-early promoter vector (pcDNA3.1) that has a bovine growth hormone polyadenylation signal (Figure 1A). The ORFs were introduced to place the gag ATG initiation codon into a consensus Kozak sequence, and all constructs were verified by DNA sequencing. The Mtv-1 gag was selected because it is plasmid-propagatable and because an infectious molecular clone (pHyb-Mtv) containing this allele exists. A putative human origin MMTV gag allele, (HBRV), that is $99.7 \%$ identical to Mtv-1 at the nucleic acid and amino acid levels, was also included. The third allele, SM, was derived from the MMTV GR strain, but was previously silently mutated [31] in the gag pp21 domain to eliminate the bacterial poison sequence. Despite discrete points of polymorphic sequence, HBRV and Mtv-1 gag do not phylogenetically segregate from one another, but both segregate from MMTV GR, the source of the SM gag allele. The level of nucleic acid identity between HBRV/Mtv-1 and SM gag is 92.7\%, whereas the amino acid identity is $95.9 \%$. The highest disparity between HBRV/Mtv-1 and SM lies within the silently mutated gag $\mathrm{pp} 21$ region. The alterations in SM result in an increase in the overall $\mathrm{G} / \mathrm{C}$ content in this region from $31.5 \%$ to $51.9 \%$, yet none of the three $\mathrm{gag}$ genes appeared more codon optimized than another as indicated by their codon adaptation indices $(\mathrm{Mtv}-1=0.137$, $\mathrm{HBRV}=0.138, \mathrm{SM}=0.151$, compared to an optima of 0.958) [32].

Gag expression constructs were transfected into HEK 293T cells and subjected to metabolic labelling followed by immunoprecipitation with a rabbit polyclonal antiMMTV CA antibody. Little or no Mtv-1 Gag or HBRV Gag was detected when expressed from CMV-based gag-only constructs; however, SM Gag was readily detected. Moreover, Mtv-1 Gag derived from the pSMtHyb provirus was also expressed to detectable levels (Figure 1B). Quantification of the levels of pulse-labeled Gag derived from the CMV vectors revealed an approximately 95-fold inhibition of these two constructs relative to SM Gag $(p<0.05)$. Expression of another MMTV Gag (Mtv-8) from the same vector was similarly restricted (data not shown). Given that Mtv-1 gag and the gag from the pSMt-Hyb provirus are identical, these results indicated the presence of a repression acting against Mtv-1 Gag ORF expression as well as a virusmediated derepression that allows Mtv-1 Gag expression from the provirus.

Since human cells are not predicted to be the natural host for MMTV replication, barriers to MMTV expression could be responsible for the inhibition of Gag expression observed. A similar inhibition has been reported in efforts to express HIV-1 Gag in murine cells [33]. Therefore, we tested whether Gag expression constructs produced protein in C57BL6 murine embryonic fibroblast (MEF) cells. We were surprised to find that both Mtv-1 and HBRV failed to express Gag whereas the pSMt-Hyb infectious molecular clone and the SM Gag variant expressed at high levels (Figure 1C, SM and pSMt-Hyb lanes). MMTV is clearly capable of replication in murine cells, and the virus is readily produced by transfection of infectious molecular clones into human cells [34-36]. Our data suggest a blockade to translation of Gag expressed in the absence of the rest of the genome in murine cells, and these data are supported by earlier reports from Vaidya et al., although the cause for this lack of expression was not determined [37].

Gag expression was also tested in two human breast cancer cell lines MCF7 and T47D and in African green monkey kidney (COS-1) cells. Similar to our data for Gag expression in HEK 293T and MEF cells, little or no HBRV or Mtv-1Gag was detected, but SM Gag expression was evident in all cell lines tested (Additional File $1)$.

\section{All gag alleles express in vitro}

Given the perplexing lack of protein expression in the CMV-gag transfection experiments, we sought to verify construct integrity and the sensitivity and capability of our polyclonal antibody to detect proteins derived from the various gag alleles. We took advantage of the fact that pcDNA3.1 vectors contain a phage T7 RNA polymerase promoter that can be used to express RNA in vitro. The in vitro synthesized RNA was then used to program in vitro rabbit reticulocyte lysate translation reactions that included $\left[{ }^{35} \mathrm{~S}\right]$-methionine. The resulting labelled protein was separated by SDS-PAGE and either visualized directly by autoradiography, or subjected to immunoblotting. All Gag constructs expressed similar levels of protein in reticulocyte lysates (Figure 1D), demonstrating that all three mRNAs are competent for programming transcription and translation in vitro. We verified these bands were indeed Gag since the product of each construct was equivalently detected by immunoblotting with polyclonal anti-MMTV CA antibody 


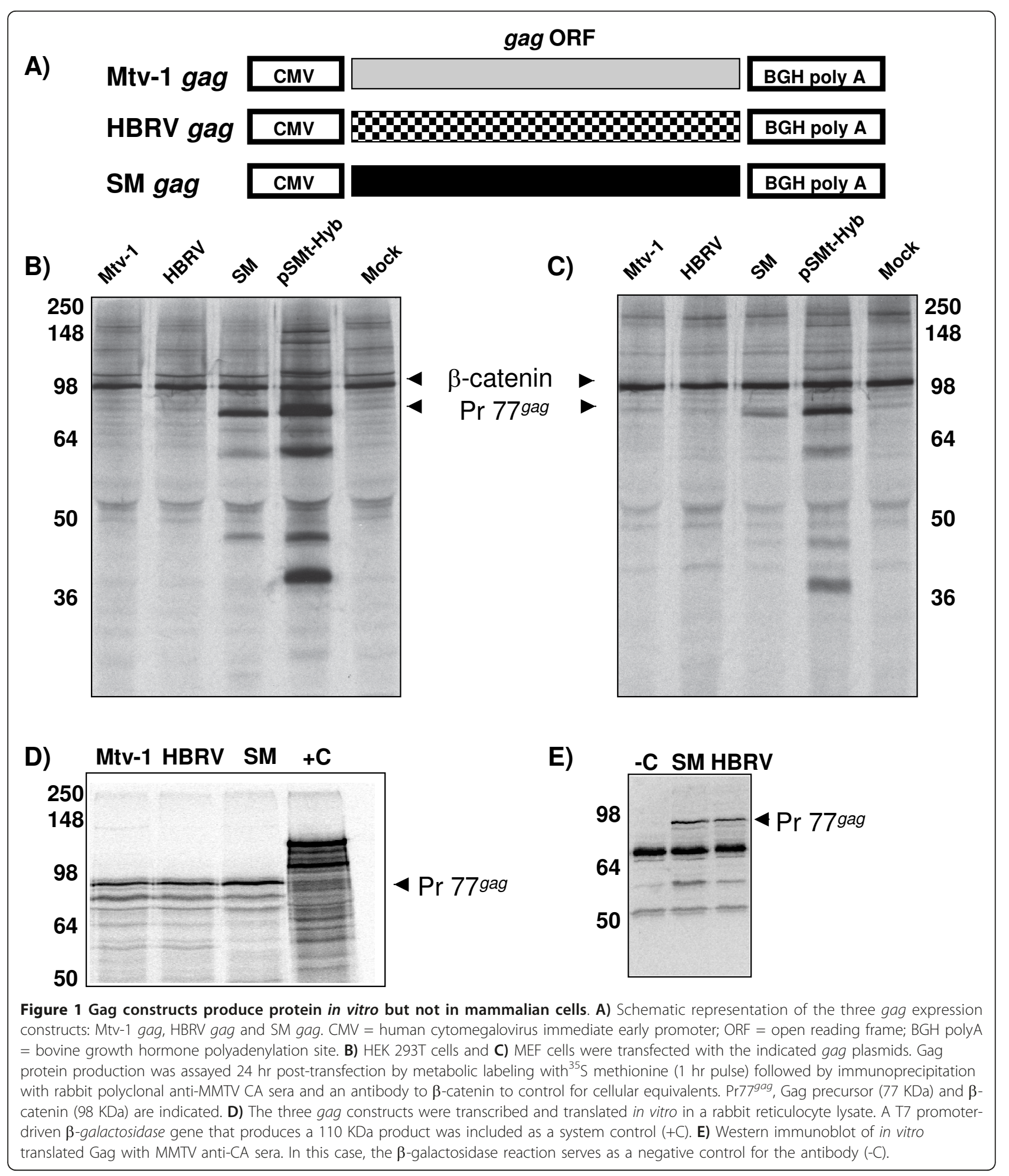


(Figure 1E). These results demonstrate that there is neither differential detection nor differential translation capacity between constructs. Taken together, these results support the concept of specific inhibition of expression of Gag dependent upon the allele present in the construct.

\section{Expression timing and protein stability do not account for lack of Gag protein}

Since all three gag-only constructs have the same promoter and would be anticipated to express with similar kinetics, we determined the expression of each at 24, 48 and $72 \mathrm{hrs}$ post-transfection by pulse labeling. SM Gag was detectable, but Mtv-1 and HBRV Gag expression was inhibited at all time points (Figure 2A). We also assessed steady-state Gag levels by immunoblotting. Only the SM Gag was detected 48 and 72 hrs posttransfection (Figure 2B). One explanation for the above results might be that Mtv-1 and HBRV Gag are highly labile proteins and therefore do not accumulate. To test this concept, we subjected CMV-gag transfected cells to metabolic pulse-labeling for 15 minutes with no chase. This time-frame was anticipated to catch synthesized protein before it could be degraded. Under these conditions, we did not detect either Mtv-1 or HBRV Gag. A robust SM Gag band was evident (Figure $2 \mathrm{C}$ ). We also used the proteasomal inhibitor MG132 (Figure 2D) or lactacystin (not shown) to block protein degradation and turnover. Proteasomal inhibition was demonstrated by a treatment-induced increase in GFP as well as

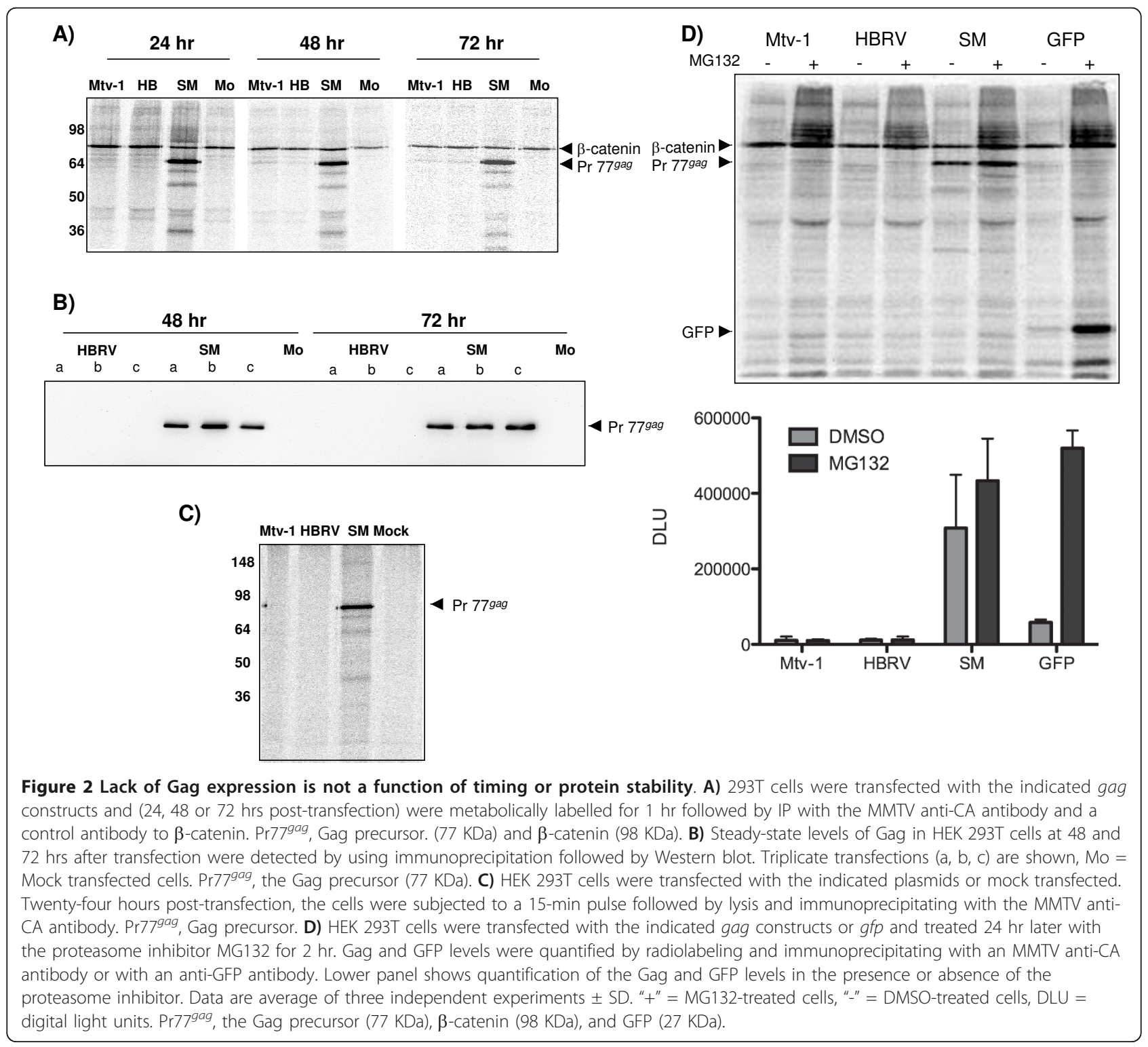


increased $\beta$-catenin signal at $98 \mathrm{KDa}$. We also noted the presence of higher molecular weight bands in the presence of the proteasomal inhibitor that likely represent accumulation of ubiquitinated and polyubiquitinated $\beta$ catenin (Figure 2D). These higher weight forms of $\beta$ catenin are also present in the GFP-only lane, and therefore, are not Gag. Despite evidence of proteasome inhibition, no Mtv-1 or HBRV Gag was detected in treated cell lysates. SM levels, however, increased in response to treatment, indicating that a substantial proportion of Gag fails to assemble, and is degraded intracellularly (Figure 2D), as has been demonstrated in other retroviral systems [38]. Thus our results show that Mtv-1 and HBRV gag constructs fail to undergo protein synthesis rather than producing a labile gene product, whereas the SM gag construct is not subject to a similar constraint.

\section{Gag transcripts are efficiently synthesized in human cells}

Our data suggest that the lack of detectable Gag results from a lack of Gag protein synthesis and not from protein degradation or instability. Therefore, the block in protein expression could result from three potential defects: the lack of gag mRNA, the lack of gag mRNA transport to the cytoplasm, or the inability to translate the Gag transcript if synthesized and appropriately transported. To identify the defect, we tested for correlations between gag mRNA levels and protein expression between constructs. Real-time PCR (qRT-PCR) was used to quantify transcript abundance, nuclear to cytoplasmic transport, and mRNA stability. We primed cDNA synthesis in transfected cells from an oligo-dT primer and performed Taqman detection using an amplicon over conserved sequences in pp21. The amplification signal $\left(C_{t}\right)$ for gag mRNA from the respective constructs was normalized to that of cellular gapdh and the reciprocal ratios were compared. We observed no significant difference in mRNA abundance for the different gag alleles (Figure 3A), indicating that all gag transcripts were present at the same level and that the lack of Gag expression was not a function of mRNA abundance.

\section{Nuclear to cytoplasmic transport and mRNA stability are similar for all gag alleles}

We next asked whether the various gag mRNAs can traffic from the nucleus to the cytoplasm. Subcellular fractionation was used to extract mRNA from nuclear and cytoplasmic fractions, and the abundance of the gag transcript in each fraction was quantified relative to gapdh by using qRT-PCR. To control for proper nuclear and cytoplasmic separation, we assessed the distribution of histone 3 and GAPDH proteins in the different fractions. We found that histone 3 was present only in the nuclear fraction while GAPDH was only in the

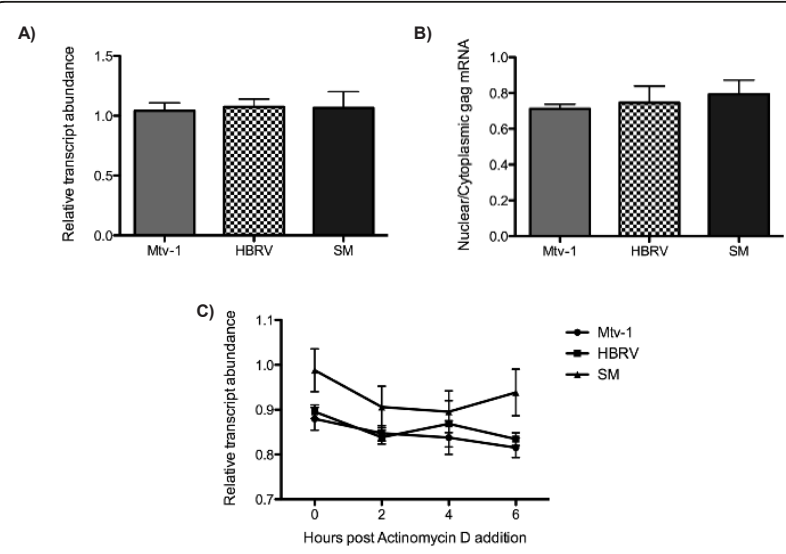

Figure 3 Inhibition of expression occurs post-transcriptionally and does not affect mRNA transport and stability. A) Total RNA was extracted from HEK 293T cells transfected with Mtv-1, HBRV, and SM gag constructs. The gag and gapdh mRNAs were reverse transcribed into CDNA using an Oligo-dT primer and quantified by Real-Time PCR. The relative abundance of gag mRNA to gapdh is calculated as the reverse of the ratio of gag CT to gapdh CT value. B) Transfected HEK 293T cells were separated into nuclear and cytoplasmic fractions. The mRNA was extracted from each fraction, and gag and gapdh mRNAs were quantified by reverse transcription Real-Time PCR. The level of mRNA transport from the nucleus to the cytoplasm was quantified as the ratio of nuclear and cytoplasmic gag mRNA relative to gapdh. C) Cells were treated with actinomycin D, and RNA was extracted from the cells at 0, 2, 4 and 6 hrs posttreatment and quantified by reverse transcription Real-Time PCR. The relative abundance of gag mRNA to gapdh was calculated at each time point. Data are the average of at least three independent experiments \pm SD.

cytoplasm (data not shown). All gag mRNAs were detected in the cytoplasmic fraction, and quantification of the ratio of the nuclear-to-cytoplasmic transcript abundance revealed no difference between gag constructs; therefore, the transport of all gag mRNAs was equivalent (Figure 3B). This result suggests that Mtv-1 and HBRV Gag expression was not impaired by a defect in mRNA transport.

Inhibited translation of HIV-1 gag has been linked to cytoplasmic instability of the RNA imparted by instability (INS) sequences in Gag [21,39]. To determine whether the various gag mRNAs displayed differential stability in the cytoplasm, CMV-gag transfected cells were treated with the transcription inhibitor actinomycin D. The gag mRNA abundance was quantified relative to gapdh by using qRT-PCR at 2, 4, and $6 \mathrm{hr}$ after treatment. No differences in mRNA stability were detected among Mtv-1, HBRV and SM over the $6 \mathrm{hr}$ treatment course (Figure 3C).

Since we determined that analyses of RNA synthesis, transport and stability do not affect the differential protein expression phenotypes evinced between SM and the two other MMTV gag alleles, we conclude that the 
block to Gag expression occurred post-transcriptionally such that the mRNA is not translatable although it was transported to the cytoplasm.

\section{Negative regulation against Mtv-1 and HBRV gag acts at the transcript level}

Post-transcriptional inhibition of Gag expression could function in two ways: (1) the inhibition directly targets the transcript for sequestration or degradation similar to that which occurs in RNAi pathways or (2) Mtv-1 and HBRV gag transcripts fail to support translation initiation. In the latter, this would be envisioned as a failure to successfully recruit or engage translation factors essential for cap-dependent translational initiation. To differentiate between these mechanisms, we introduced gag alleles into a bicistronic plasmid vector in which the Gag translation is driven by cap-dependent initiation and eGFP is translated from an encephalomyocarditis virus- internal ribosome entry site (EMCV-IRES) (Figure
4A). We reasoned that if inhibition was at the level of gag translation initiation, then no Gag would be produced from cap-dependent initiation; but the production of eGFP from the cap-independent IRES would proceed unabated. However, if the inhibition acts to prevent all interactions of the mRNA with translational machinery, then neither cistron would be expressed.

Bicistronic constructs were transfected into HEK293T cells and the transfected cultures were observed by in situ fluorescent microscopy for eGFP expression prior to lysis and immunoprecipitation to quantify MMTV Gag expression. In a parallel analysis, we used qRT-PCR to demonstrate that all bicistronic constructs transported mRNA to the cytoplasm (data not shown). We observed no eGFP signal from the bicistronic construct containing HBRV gag, and immunoprecipitation revealed no detectable Gag from the first cistron of this construct (Figure $4 \mathrm{~B}$ and $4 \mathrm{C}$ ). In contrast, for the SM bicistronic construct both proteins were abundantly

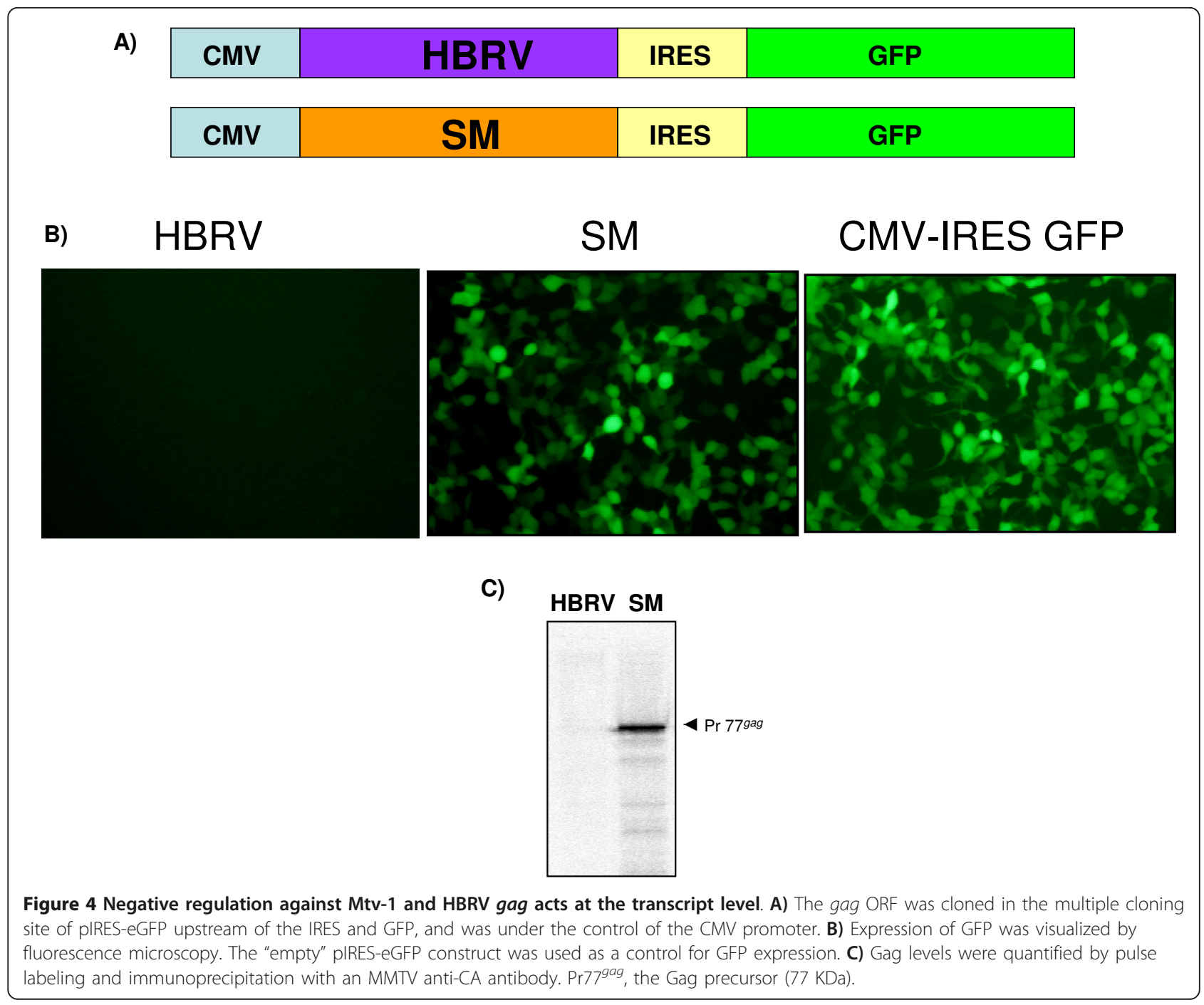


produced (Figure 4B and 4C). Since both constructs contained the same Kozak sequence and IRES, the inhibition resulted from recognition of sequences within gag and not from a defect in translational initiation. Thus, gag sequences appear to either inhibit translational 'licensing' of the mRNA or to completely sequester the mRNA away from translational machinery.

\section{Inhibition is dependent on the nuclear history of the mRNA}

Analysis of bicistronic constructs suggested that the gag mRNA was not competent for translation (Figure 4), yet all three gag mRNAs can program a rabbit reticulocyte lysate to make protein (Figure 1D). We, therefore, tested whether in vitro synthesized gag RNAs were competent to program translation if delivered directly to cells by transfection. This approach was also reasoned to provide some capacity to distinguish between cytoplasmic or nuclear mechanisms of translational inhibition. The pcDNA3.1 constructs contain a T7 RNA polymerase promoter that was used to drive RNA synthesis in vitro. A portion of each in vitro synthesized gag RNA was used to program a rabbit reticulocyte lysate to test RNA integrity and the remainder of the same preparation was introduced into HEK293T cells using lipid mediated transfection. In contrast to transfection of plasmid pCDNA3.1 gagconstructs (Figure 5A and 5C), the in vitro synthesized gag RNAs (derived from the same construct) were fully competent to program translation in

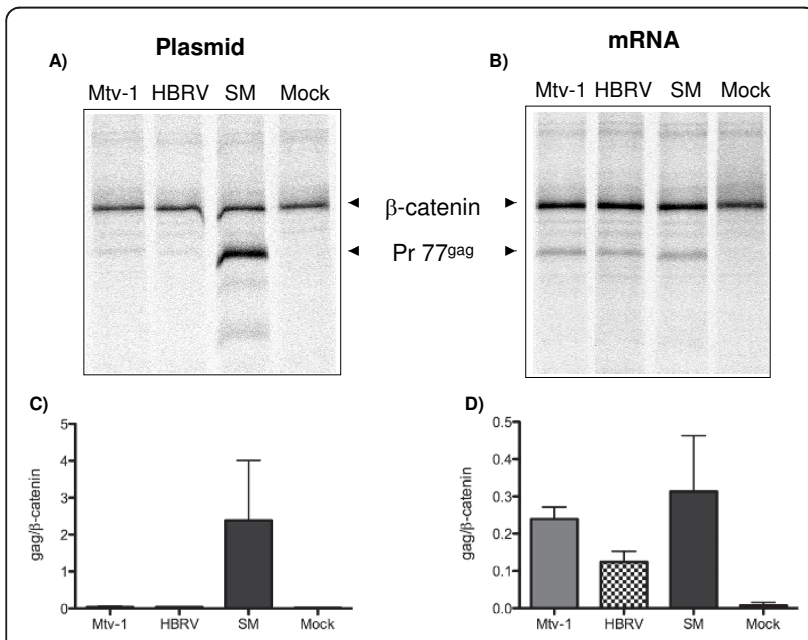

Figure $\mathbf{5}$ Inhibition is dependent on the nuclear history of the mRNA. A) Plasmid or B) in vitro made mRNA for the indicated constructs was transfected into HEK $293 \mathrm{~T}$ cells. After 24 hr, Gag expression was quantified by pulse labeling for $1 \mathrm{hr}$ and immunoprecipitating with an MMTV anti-CA antibody. $\beta$-catenin immunoprecipitation served as a loading control. Gag expression from plasmid $\mathbf{C}$ ) or in vitro made mRNA D) was quantified relative to $\beta$-catenin. Data represent the average of three experiments \pm SD Pr779ag, the Gag precursor (77 KDa) and $\beta$-catenin (98 KDa). the RNA transfected cells (Figure 5B and 5D). While we do not formally know whether the introduced RNA remained cytoplasmic or trafficked to the nucleus, these results suggest that inhibition of Mtv-1 and HBRV Gag translation was imposed in the nucleus or acts cotranscriptionally.

\section{Rescue of Gag expression by the RmRE and Rem in trans} is dependent on splice recognition sites

Expression of MMTV gag from a proviral construct is readily detected while the same reading frame is inhibited in a $g a g$-only construct. This suggests an inhibition targeting gag ORF and a viral mechanism to overcome it. We have shown that this inhibition targets the mRNA in the nucleus and prevents its translation in the cytoplasm. From studies using other retroviruses, there are two potential viral mechanisms that rescue Gag expression from inhibition. The first, as exemplified by spleen necrosis virus and Mason-Pfizer monkey virus, involves an interaction between cellular RNA helicase A and the viral 5' UTR that enhanced viral mRNA association with ribosomes $[24,25]$. The second is post-transcriptional enhancement of HIV-1 translation by the viral Rev protein [40].

\section{The MMTV LTR and 5'UTR sequences are not sufficient to} rescue Gag expression

The natural promoter for MMTV Gag is in the 5'LTR and requires glucocorticoid stimulation for activity [41]. While expression from the LTR is typically lower than from CMV promoter-driven over-expression systems, testing whether addition of glucocorticoid (dexamethasone) rescued expression was deemed worthwhile. Moreover, given reports that the presence of an authentic 5' UTR can be important for regulating retroviral expression [42], and that the 5'-UTR can enhance translation of the gag gene [25], we created an MMTV gag construct driven by its own LTR. We deleted pol and part of the env gene from pHyb-Mtv while maintaining the 3'LTR. The resultant construct, pLTR-gag (Figure 7A), contains the 5' and 3' LTRs, Mtv-1 gag, pro, the $R m R E$ and sag. Gag expression from this construct was reduced compared with that from the intact provirus (Additional File 2). This result suggests that having all of the known 5' cis-sequences, the RmRE and the 3' LTR from the provirus fails to support expression of Gag at levels similar to an intact proviral construct containing the same gag ORF. Moreover, addition of dexamethasone had no impact on Gag expression except from the intact provirus. Thus the 5'-UTR appears to be insufficient to rescue Gag expression.

Rem and RmRE are insufficient to rescue CMV-driven Gag expression

The gag expression constructs presented thus far do not contain known splice sites and thus would be 
anticipated to be independent of the RNA transportmediated activities of Rem. Our data quantifying nuclear and cytoplasmic gag mRNA levels support this concept. However, several reports have suggested post-transcriptional roles for Rev and its analogs that potentially impact Gag protein expression [15,21]. These data prompted us to test whether the observed restriction against Gag expression results from the lack of Rem or the lack of a Rem/RmRE interaction.

We began by verifying the functionality of three previously published Rem constructs (Rem-only, GFP-Rem, and GFP-RemSP) [13] by testing their capacity to mediate expression of an intronic Renilla luciferase reporter from pHMRluc, a construct that also contained the
RmRE (Additional File 3). We tested whether providing Rem in trans altered expression from the CMV-gag only constructs lacking a RmRE (not shown) and observed no effect. Thus, the silent alterations in SM gag did not introduce a Rem responsive cis-element. Next, we cloned the RmRE sequence (nt 7291-7886) into the 3' UTR of the CMV-driven gag constructs (Figure 6A). For a precise location of the RmRE sequence compared to the $M t v-1$ genome and relative to the published RmRE see Additional File 4. After verifying that no extraneous mutations were introduced in $g a g$, we demonstrated that the construct expression profiles remained unchanged from those observed in the absence of the RmRE (Figure $6 \mathrm{~B}$ compare lanes ' 1 ' to Figure $1 \mathrm{~B})$. However, co-

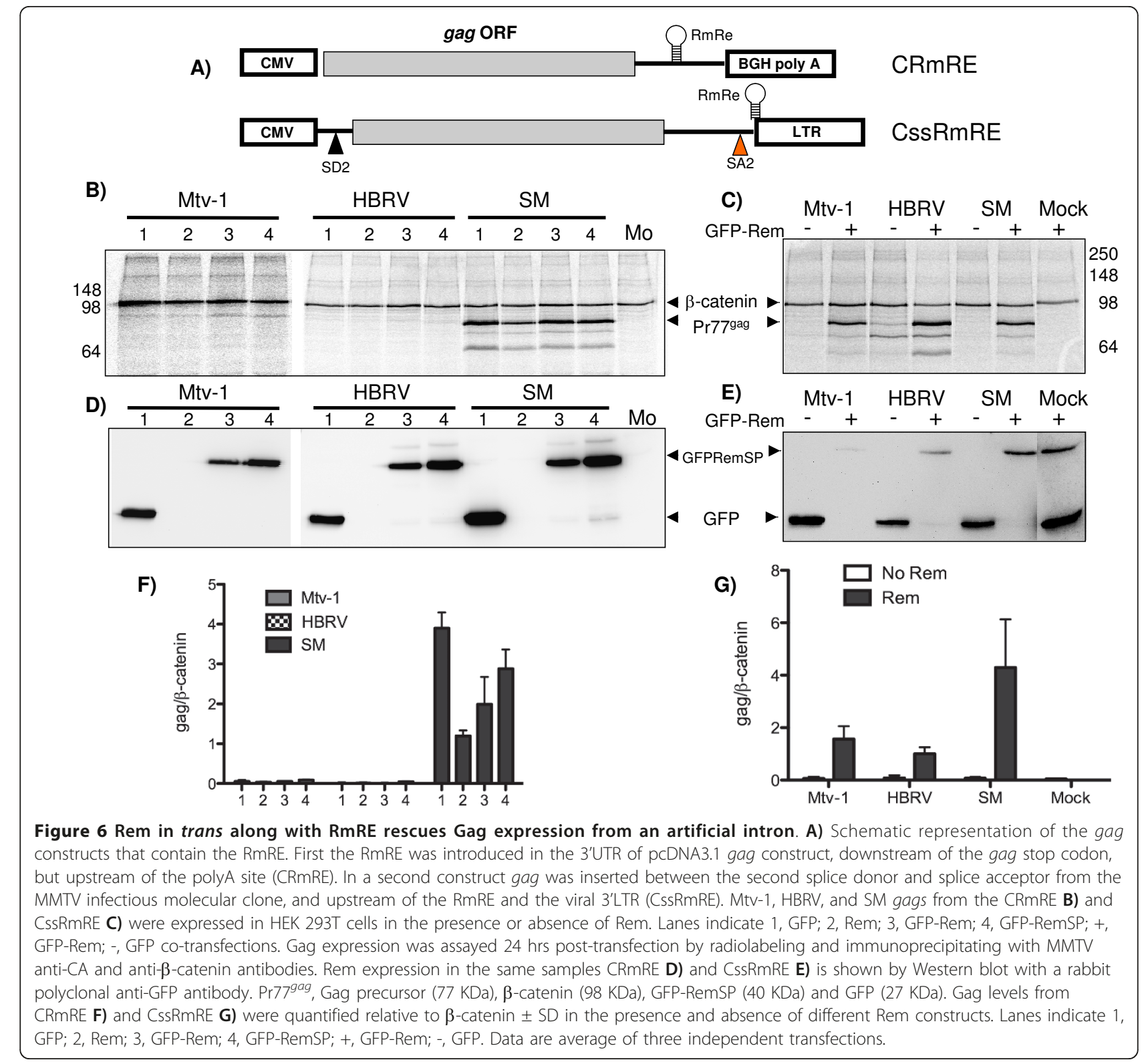


transfection of Rem did not rescue Gag expression despite the presence of the RmRE (Figure 6B and 6F; lanes 2, 3, and 4). In Figure 6D, anti-GFP immunoblotting was utilized to demonstrate expression of the two Rem fusion constructs provided in trans. Moreover, no difference in the levels of cytoplasmic mRNA between gags expressed in the presence or absence of Rem was detected. This result suggested that providing Rem in trans did not alter nuclear-to-cytoplasmic transport of these gag mRNAs despite the presence of its cognate RmRE cis sequence.

Rem in trans along with RmRE rescues Gag expression from an artificial intron

When expressed from the viral LTR, both the genome length gag/gag-pro-pol mRNA and the subgenomic, singly-spliced env mRNA are contained within introns. Yet the cellular default is to completely splice pre-mRNA before transport to the cytoplasm. Like other complex retroviruses, MMTV encodes a trans-acting, Rev-like regulatory protein, Rem, which in combination with the RmRE, is necessary for expression of intronic viral gene products $[13,18]$. We replaced Renilla luciferase in pHMRluc gene with the three gag genes. In this context, gag is in the intron and should not be expressed unless splicing is overcome by a transport regulator. As anticipated, no Gag expression was detected from this construct in the absence of Rem (Figure 6C and 6G '-' lanes). However, with Rem in trans, equivalent Gag expression was evident for all three constructs (Figure $6 \mathrm{C}$ and $6 \mathrm{G}^{\prime}+$ ' lanes). Figure $6 \mathrm{E}$ shows immunoblots of the GFP-Rem fusion proteins and controls. Results from these investigations demonstrate that Rem can rescue Gag expression but only when gag is flanked by a splice donor and acceptor, and the mRNA contains the RemRE.

\section{Rem along with the RmRE and natural splice donor and acceptor sequences rescues Gag expression}

Based on the results demonstrating Gag expression from an artificial intron RmRE construct, we revisited the MMTV pLTR-gag construct where Rem failed to rescue Gag expression despite the fact that pLTR-gag contained an intact RmRE (Figure 7B and 7F and Additional File 4). As in Figure 6, immunoblotting for GFP was utilized to demonstrate expression of the GFP-Rem fusion protein (Figure 7D and 7E). Although the major splice donor was present in pLTR-gag, both viral splice acceptors had been deleted (Figure 7A). Upon reconstruction of the splice acceptor into the pLTR-gag construct (pLTR-gagSA), Rem was able to rescue Gag expression (Figure 7C and 7G and Additional File 4). This result further supports the concept that Rem requires, in addition to the RmRE, the recruitment of splicing machinery to gag intron splice donor and acceptor sites, yet such recruitment does not result in cleavage of the intron.
This complex of cis and trans factors is necessary to support MMTV Gag translation.

\section{Discussion}

Nuclear protein-mRNA interactions impact viral RNA transport. The transport mechanisms (Crm1 or Tap/ NXT), in turn, influence the localization and fate of the viral mRNA or gRNA in the cytoplasm. These relationships are evident from studies of RSV in which deletion of a direct repeat upstream of $s r c$ that functions as a CTE leads to efficient Gag production but lack of particle assembly $[43,44]$. Similar defects are observed in attempts to express ALV in mammalian cells [45-47], but the defect can be overcome by driving ALV through the Crm1 RNA export pathway by providing an HIV-1 RRE and Rev in trans [48]. HIV-1 Gag expression can be achieved in murine cells, but processing, viral RNA encapsidation, and particle formation are not supported unless the HIV-1 RNA is exported via a CTE export pathway [33]. Murine expression of HIV-1 Gag can be achieved in a Rev/RRE/Crm1-dependent pathway but only if human SRp40 and SRp55 are provided ectopically [49]. In addition, post-transcriptional control elements (PCE) in the 5' UTR of a number of retroviruses have been shown to interact with nuclear or cytoplasmic RNA helicase A to promote mRNP remodeling that facilitates polyribosome association resulting in efficient translation [26,42]. Thus protein marks applied to the pre-mRNA in the nucleus, in addition to defining the RNA export pathway, contribute to localization and fate of the RNA in the cytoplasm.

From the studies mentioned above, as well as from our CMV-gag expression data (Figure 1), it is clear that mRNA export alone is insufficient to stimulate translation of retroviral Gag. All alleles of MMTV gag that were tested with the CMV promoter were efficiently exported, presumably by a Tap/NXT pathway, yet differential Gag translation occurred among the alleles. We propose that RNA 'fate' determinants are being applied to MMTV gag mRNAs that are dependent upon sequences within the ORF. These RNA fate decisions are potent enough to prevent cap-independent translation of reporters in the second position of bicistronic constructs, suggesting that nuclear 'marks' applied to the mRNA and direct its cytoplasmic localization such that it is sequestered from all translation machinery. Yet, the RNA is not degraded.

Rev controls the transition between early and late phases of HIV-1 replication by regulating the export of incompletely spliced viral mRNA to the cytoplasm. While promoting unspliced mRNA export is regarded as its primary function, Rev has also been shown to affect the stability, translation and encapsidation of RRE-containing RNAs $[40,50]$. Rev has been shown to overcome 


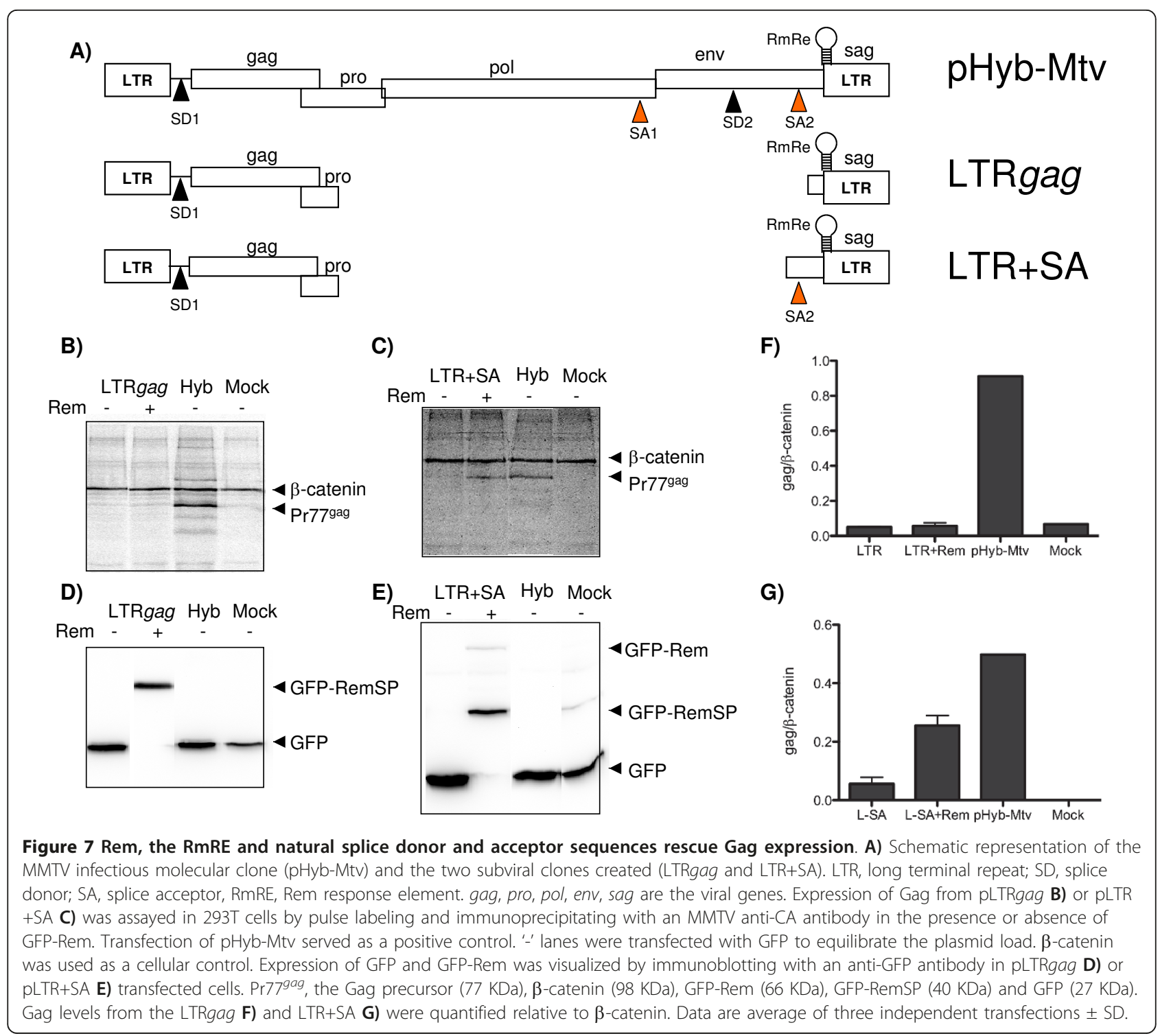

inhibitory or instability (INS) sequences present in the gag ORF [21] by preventing cytoplasmic RNA degradation. The gag RNA can also be protected from degradation and efficiently expressed if it is codon optimized or if the INS sequences are mutated [39]. The fact that silent mutations in MMTV SM gag overcome inhibition of expression acting against Mtv-1 suggests that wildtype MMTV gag genes may contain an INS. However, in contrast to the HIV-1 inhibitory sequences, which trigger the viral RNA for degradation, all MMTV gag mRNAs were stable in the cytoplasm whether translated or not. Moreover, our analyses of the three MMTV gag alleles did not reveal evidence of codon optimization, or lack thereof, by any particular gag. Thus, we concluded that MMTV gag sequences were subject to post-transcriptional control mechanisms, but they function differently from the mRNA degradation pathways that act against HIV-1 gag. HIV-1 INS sequences were bound in the nucleus by PSF, a transcription/splicing factor, which in turn affected the fate of the RNA in the cytoplasm [51]. It remains to be tested whether a similar interaction dictates the fate of the MMTV gag alleles.

Like HIV-1, MMTV has been characterized as a complex retrovirus due to the presence of the Rev analog, Rem, that temporally controls MMTV gene expression [13]. Rem has been shown to interact with a structured element in unspliced and singly-spliced mRNAs [14] and to promote transport of these RNAs to the cytoplasm [13,18]. Mertz et al. demonstrate that Rem enhances translation of an intronic reporter without affecting RNA transport [52]. Moreover, Hofacre et al. have shown that the function of JSRV Rej appears to be 
involved in cytoplasmic enhancement of translation not in nuclear-to-cytoplasmic RNA transport [15]. In our hands, gag bicistronic constructs were efficiently transported to the cytoplasm. If cap-dependent translation was inhibited by the gag allele in the first cistron, no reporter was expressed by cap-independent translation from the second cistron. Thus, the RNA has been targeted by an inhibition that manifests as a total and irreversible block to translation. This inhibition is likely a function of RNA localization as opposed to failure to initiate translation since IRES-mediated translation is also inhibited. These results reinforce the concept that it is the gag sequence itself that is the target of inhibition since the eGFP reporter is efficiently co-expressed when SM Gag is expressed. Moreover, direct RNA transfections reveal that the inhibition is nuclear in origin since all gag alleles were equally competent to program translation. These allele-specific effects on Gag translation are summarized in Figure 8. We reasoned that the directly transfected RNA either does not have access to the nucleus or it escapes a binding partner that is cotranscriptionally acquired. While our data are consistent with that of others demonstrating a phenotypic translational block, we show that this blockade is actually imposed as a nuclear mark on the RNA.

The nucleotide sequences among complex retroviruses are not highly conserved. It is therefore not surprising that there is little consensus in RNA regulatory mechanisms other than a requirement for a cis regulatory element (like the RmRE) and a cognate trans-RNA-binding factor (like Rem). Other sequences in the RNA may be essential because of their capacity to specifically coordinate nuclear proteins. In the elucidation of Rem and Rej function, reporter constructs that contain splice sites have been used to assay effects on RNA transport $[13,15]$. However, in the absence of Rem and Rej the intron-containing constructs were exported, but not expressed. The interpretation was that the function of Rem and Rej was to enable translation rather than RNA transport. The potential role of the splice sites and their capacity to coordinate nuclear proteins that impact mRNA fate was not directly addressed. We have shown that the presence of splice sites, but not their utilization, is required in addition to the RmRE and Rem for Gag expression. This is potentially due to interaction of these sequences with protein partners as has been shown in HIV-1 where U1 snRNP interaction with the 5 ' splice site is required for Rev function [53]. However, we show that the 5 ' MMTV splice site alone is insufficient to support efficient export and translation. Similarly, any combination other than a functional splice donor and acceptor with a functional Rem and RmRE interaction is insufficient. Figure 8 shows our conceptual model of Gag translation competence. Retroviral RNAs have been demonstrated to interact with a variety of proteins that are constituents of RNA splicing transport and localization complexes, including hnRNPA1, hnRNP A2, I/PTB, DDX1, DDX3, RHA, nucleolin, Sam68, ASF/ SF2, SRp40/p55, EF1 $\alpha$, Pur1 $\alpha$, and Staufen [54-56]. We conclude that MMTV Gag expression requires splicing sites on the mRNA in addition to viral RNA regulatory components. Yet while these interactions are essential to facilitate the cytoplasmic expression of Gag, they do not function normally since the intron is not removed. It is possible that Rem is participating in direct and perhaps inhibitory interactions with splicing components that are important for transport or RNA localization and utilization or perhaps the virus has adapted to subvert nuclear RNA-binding protein function in order to achieve its expression program. Thus, it is clear that in the presence of a complete set of viral cis signals as well as the viral Rem protein, MMTV gag RNA is exported through Crm1 [13], and through these signals, allelespecific inhibitory mechanisms are countered making gag competent for translation. Our findings suggest that continued investigation of the proteins that differentially interact with Mtv-1 and SM gag alleles may enlighten our understanding of how nuclear mRNP interactions participate in regulating RNA localization and fate.

\section{Conclusions}

Nuclear interactions inhibit MMTV gag gene expression independent of RNA transport, stability, and translation. To overcome the inhibition and achieve translation of MMTV gag mRNA in the cytoplasm, both the Rem protein and its cognate binding target the RmRE, and surprisingly, a splice donor and acceptor sequence are required.

\section{Methods}

\section{Cell culture and transfections}

Human embryonic kidney (HEK 293T) cells, and murine embryonic fibroblasts (MEF) were maintained in Dulbecco's Modified Eagle Medium supplemented with 10\% fetal bovine serum, L-glutamine $(20 \mathrm{mM})$, penicillin $(100 \mathrm{U} / \mathrm{mL})$ and streptomycin $(100 \mu \mathrm{g} / \mathrm{mL})$. Cells were grown to $60-80 \%$ confluency in $60 \mathrm{~mm}$ plates and were transfected with $5 \mu \mathrm{g}$ of the indicated plasmid DNA at a 1:3 ratio of DNA to FuGene 6 (Roche), following the manufacturer's instructions and were assayed $24 \mathrm{hr}$ post-transfection, or as indicated.

\section{Plasmid constructs}

All restriction enzymes were purchased from New England Biolabs, and $P f u$ polymerase was acquired from Stratagene. The hybrid MMTV provirus (pHyb-Mtv) (graciously provided by Jackie Dudley Ph.D.) [28] and pSMt-HYB [57], which is an MMTV infectious 


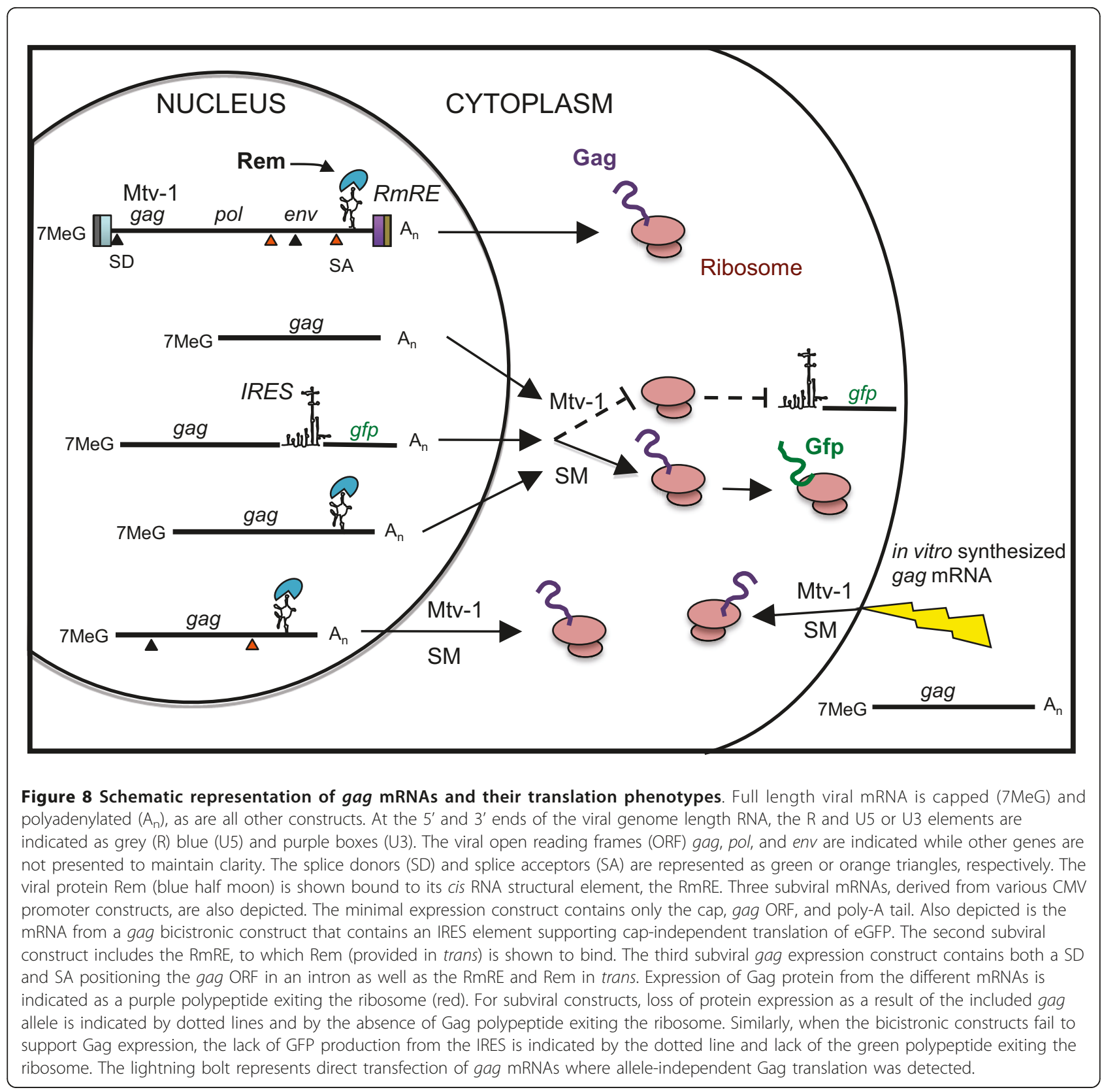

molecular clone driven by the Mason-Pfizer monkey virus LTR were used as positive controls. The gag sequences utilized as templates for amplification, mutagenesis and expression were $M t v-1$ (accession \# AF228550), MMTV GR [58-60] and HBRV [61]. Sequences encoding the Gag ORF were PCR amplified using primers: Forward: 5'-ACCATGGGGGTCTCGG-3' and Reverse: 5' - TTACAAGTTTTTTGAATTTTCGG3'. For HBRV gag, the template DNA was amplified by PCR 'stitching' of three patient-derived template fragments,: 174-1 (accession \# AF513918), 175-3 (accession \# AF513919) and 168-2 (accession \# AF513914) [61].
The pHyb-Mtv plasmid was used as a source for Mtv-1 gag and the silently-mutated (SM) MMTV GR gag from the pDAB-MMTV plasmid, which allows plasmid propagation [31], was the source for SM gag. Amplified products were TA-cloned into pCR2.1 TOPO (Invitrogen) and then transferred into the eukaryotic expression vector pcDNA3.1 (Invitrogen) by EcoRI restriction digestion and ligation. The orientation of the gene in pcDNA3.1 was determined by $S t u \mathrm{I}$ restriction digestion, and correctly oriented clones were sequenced across the reading frame to assure no unforeseen mutations occurred during PCR cloning. 
The RmRE sequence [14,19] was PCR amplified with the primers: Forward: 5'-TTTGATATCCATTGTTTTCCAGTGCCTTGC-3' and Reverse: 5'-TTTCTCGAGCT CTTTCTATTTTCTATTCCCATTTC-3' and introduced into the EcoRV and XhoI sites of the pcDNA gag constructs. The plasmids pHMRluc, Rem, RemGFP and RemSPGFP were a gift from Dr Jackie Dudley [13]. To introduce the gag ORFs in the pHMRluc construct, Renilla luciferase was excised by PCR, and the XmnI site reconstituted. The three gag ORFs were introduced by digestion and blunt ligation from pcDNA3.1 constructs.

pLTR-gag was derived from pHyb-Mtv by excision of the sequence present between the two BglII sites. To introduce the splice acceptor (SA) site back into the pLTR-gag, the sequence spanning the SA (nt 6993-7384) was PCR amplified from pHyb-Mtv using primers that contain the BglII site (Forward: 5'- AGGAGATCTGCAAATTATGATTTTATCTGCG- 3' Reverse: 5' - CG GCATTTCCCCCTTTTTTC-3'). After PCR, the sequence was introduced into pLTR-gag by restriction digestion and ligation. To create bicistronic constructs the three gags were cloned in the multiple cloning site (EcoRI) of pIRES2-EGFP (Clonetech).

\section{Immunoprecipitation and Western blot}

Cells were washed 3 times with PBS and labeled for $1 \mathrm{hr}$ with ${ }^{35}$ S Cys/Met trans-label (MP biomedicals) at 100 $\mu \mathrm{Ci} / \mathrm{mL}$ in Cys/Met-free medium. Immunoprecipitations were performed as described [62]. Briefly, cells were lysed in Lysis Buffer A $(0.15 \mathrm{M} \mathrm{NaCl}, 50 \mathrm{mM}$ Tris- $\mathrm{HCl}$ (pH 7.5), 1\% Triton $\mathrm{X}-100$ and $1 \%$ deoxycholate), the nuclei were removed by centrifugation at $14,000 \times \mathrm{g}$ for $1 \mathrm{~min}$, after which SDS was added to $0.1 \%$ final concentration. Gag proteins were precipitated with $3 \mu \mathrm{L}$ rabbit polyclonal anti-MMTV CA antibody and $25 \mu \mathrm{L}$ formalin-fixed Staphylococcus aureus. Immunoprecipitated proteins were separated on 10\% SDS-PAGE followed by quantitative phosphorimager analysis. To normalize for quantification, we immunoprecipitated in parallel with an anti- $\beta$-catenin antibody (AC-15) (Sigma). The same anti-MMTV CA antibody was used for Western blots. To detect Rem-GFP fusions and GFP we used a rabbit polyclonal anti-GFP antibody (Sigma).

\section{In vitro transcription and translation}

To synthesize proteins in vitro we used the STP3 kit (Novagen) following the manufacturer's instructions. To program mRNA synthesis, $500 \mu \mathrm{g}$ of plasmid containing the T7 promoter (pcDNA3.1 Gag or controls) was introduced into a T7 polymerase transcription mixture. The reticulocyte lysate was added to the transcription reaction to translate protein in the presence of ${ }^{35} \mathrm{~S}$ -
Methionine. A $\beta$-galactosidase plasmid provided with the kit was used as a positive control.

\section{Protein stability}

HEK 293T cells were transfected as described. After 24 hours the cells were pulsed for 15 minutes with 100 $\mu \mathrm{Ci}^{35} \mathrm{~S}$ trans label, and the proteins were immunoprecipitated and analyzed by Western blotting with the antiMMTV CA antibody.

Twenty-four hours after transfection, parallel cultures of HEK293T cells were treated with $20 \mu \mathrm{M}$ MG132 (Sigma) or an equivalent volume of DMSO for 2 hours to block proteasomal degradation. Concomitantly, cells were metabolically labelled with $100 \mu \mathrm{Ci} / \mathrm{mL}^{35} \mathrm{~S}$ Cys/ Met trans-label (MP biomedicals) in Cys/Met-free medium. After two hours of treatment and labelling, protein accumulation was quantified as described previously.

\section{RNA quantification}

RNA was extracted using Trizol reagent (Invitrogen) following the manufacturer's instructions. Carry-over DNA was digested with TurboDNase (Ambion) $1 \mathrm{hr}$ at $37^{\circ} \mathrm{C}$. For cDNA production, $5 \mu \mathrm{g}$ of RNA were used to template SuperScript III reverse transcription (Invitrogen). RNA abundance was quantified by real-time PCR using Taqman technology on an ABI 7500 Fast Real-time PCR System. Briefly, $20 \mu \mathrm{L}$ reaction volumes contained $10 \mu \mathrm{L}$ of $2 \times$ Reaction Mixture (Ambion), $1 \mu \mathrm{L}$ primer and probe mix, $2 \mu \mathrm{L}$ of the template cDNA and $7 \mu \mathrm{L}$ water. Standard thermal cycling and data collection parameters were utilized. Amplification of gag cDNA was normalized to gapdh. Gag primers were forward: 5'-TGAAGAAAAGGAGAAGGCA GA-3', and reverse: 5'CTCAGGGGACAGGTCATCAT-3' and the probe was 5'-Fam-AAGGCCTTTTTA GCCACAGATTGG-Tamra3' and GAPDH primers were primer forward: 5'GAAGGTGAAGGTCGGAGTC-3', and reverse: 5'GAAGATGGTGATGGGATTTC-3' and probe was 5'JOE-CAAGCTTCCCGTTCTCAGCC-Tamra -3'.

\section{Nuclear-cytoplasmic extracts}

HEK 293T cells were partitioned into nuclear and cytoplasmic fractions using a Cell Fractionation Buffer (PARIS kit, Ambion). RNA was extracted and quantified as described above. mRNA transport was measured as the nuclear to cytoplasmic gag mRNA ratio relative to gapdh as measured by using qRT-PCR of cDNA.

\section{RNA stability}

Transcription was blocked by addition of $5 \mu \mathrm{g} / \mathrm{mL}$ actinomycin D. RNA was extracted at $0,2,4$ and $6 \mathrm{hr}$ after treatment and quantified by using qRT-PCR as described above. The relative transcript abundance at 
each time point was calculated as the ratio of gag CT to gapdh CT.

\section{Fluorescence microscopy}

To visualize eGFP expressed from the bicistronic constructs, in transfected HEK 293T cells we used an Olympus IX70 microscope with a DP70 camera at a magnification of $20 \times$.

\section{In vitro synthesized mRNA}

To make capped mRNA in vitro we used the mMessage mMachine system (Ambion) following manufacturer's instructions. The mRNA was first verified in vitro to support translation. Equivalent amounts of mRNA were then transfected into 293T cells using Lipofectamine 2000 (Invitrogen). Gag expression was assayed as described above by pulse labeling and immunoprecipitation.

\section{Additional methods}

The human breast cancer cell lines T47D and MCF7 were grown in RPMI-1640 Medium and Eagle's Minimum Essential Medium, respectively, and supplemented with $10 \%$ fetal bovine serum, L glutamine $(20 \mathrm{mM})$, penicillin $(100 \mathrm{U} / \mathrm{mL})$, streptomycin $(100 \mu \mathrm{g} / \mathrm{mL})$ and $0.01 \mathrm{mg} / \mathrm{mL}$ bovine insulin. COS-1 cells were grown in DMEM.

\section{Luciferase assay}

Cells were seeded at $5 \times 10^{4}$ per well in 24-well plates, then transfected with $2 \mu \mathrm{g}$ of the appropriate plasmid DNA using Fugene 6 (Roche) at 1:3 DNA:Fugene ratio. Twenty-four hrs after transfection, the cell lysates were assayed for luciferase activity using the Renilla Luciferase Assay System (Promega) following manufacturers instructions. Luminescence was integrated over $1 \mathrm{sec}-$ ond with a 2-second delay on a PerkinElmer 1420 Multilabel Counter Victor3V. Each transfection was done in triplicate and each lysate was assayed in triplicate.

\section{Additional material}

Additional file 1: (PPT) Similar Gag expression profiles are seen in several cell types. The indicated gag constructs were transfected into COS-1 cells, and the human breast carcinoma cell lines, T47D and MCF7. After $24 \mathrm{hr}$ Gag expression was assayed by metabolic labeling followed by immunoprecipitating with anti-MMTV CA antibody. +C, pSMt-HYB. Pr77 $7^{9 a 9}$, the Gag precursor $(77 \mathrm{KDa})$ is indicated by the arrow.

Additional file 2: (PPT) The cognate 5' UTR does not rescue Gag expression. MMTV Gag was expressed in HEK 293T cells either from an intact provirus (pHyb-Mtv) or from the MMTV pLTR-gag construct in the presence or absence of dexamethasone (Dex). After 24 hrs,

dexamethasone was added to the cells and 48 hrs post transfection, cells were radiolabeled and Gag expression was assayed by

immunoprecipitating with anti-MMTV CA antibody. Pr779ag, the Gag precursor $(77 \mathrm{KDa})$.
Additional file 3: (PPT) Rem enhances expression of a reporter gene. To verify Rem function, we used the reporter plasmid pHMR/uc in which Renilla luciferase is in an intron, upstream of the Rem response element. pHMR/uc was cotransfected with the indicated plasmids and luciferase activity was integrated over one second. The average of triplicate luciferase readings of three independent transfections \pm SD is shown. A plasmid expressing Renilla luciferase from a CMV promoter was used as a positive control (Renilla) and mock-transfected cells were used as a negative control. The GFP lane shows the basal luciferase expression from the pHMR/uc plasmid.

Additional file 4: (PPT) Location of RmRE elements in constructs The viral genomic RNA from nt 6709-8564 in Mtv-1 (see Methods for accession \#) is shown on the top line with the splice acceptor 2 (SA2) indicated by the orange triangle and the initiation and termination codons of Sag and Env. The two published RmRE sequences are depicted relative to their position versus the reference genome. Following the published RmRE sequences are those utilize in the current manuscript beginning with the sequence that was introduced into the CMV promoter Gag-only constructs (CRmRE) and the same construct containing splice donor and acceptor sequences (CssRmRE). The final two sequences are those that were introduced into proviral derived Gag expression constructs that lacked the splice acceptor (LTRgag) or contained a functional splice acceptor (LTR+SA). For clarity, promoter and gag sequences $5^{\prime}$ to the area introduced to contain the RmRE and splice sites are not shown.

\section{List of abbreviations}

MMTV: mouse mammary tumor virus; ORF: open reading frame; RmRE: Rem response element; mRNP: messenger ribonucleoprotein; $M L V$ : murine leukaemia virus; CTE: constitutive transport element; HIV-1: human immunodeficiency virus; LTR: Iong terminal repeat; JSRV: Jaagsiekte sheep retrovirus; INS: instability or inhibitory sequence; PCE: post-transcriptional control element; SM: silently mutated; HBRV: human betaretrovirus; CMVcytomegalovirus; MEF: murine embryonic fibroblasts; COS-1: African green monkey kidney cells; HEK 293T: human embryonic kidney cells; qRT-PCR: quantitative reverse-transcription polymerase chain reaction; ECMV-IRES: encephalomyocarditis virus internal ribosome entry site; SD: splice donor; SA: splice acceptor.

\section{Acknowledgements}

The authors would like to thank Jaquelin Dudley at the University of Texas at Austin for providing suggestions and DNA constructs. We thank Gillian Air and David Dyer for helpful discussions related to interpretation of the data and for critical reading of the manuscript. We also would like to thank Tina Blader for assistance in manuscript preparation. This publication was made possible by Grant Number P20RR016478 from the National Center for Research Resources (NCRR), a component of the National Institutes of Health $(\mathrm{NIH})$.

\section{Authors' contributions}

IB conducted all experiments, assisted in data interpretation, and prepared the draft version of the manuscript. MS participated in early phases of experimental conceptualization and assisted in data interpretation. JW developed the experimental plan and data interpretations as well as assisting in manuscript preparation and editing. All authors read and approved the final manuscript.

\section{Competing interests}

The authors declare that they have no competing interests.

Received: 6 December 2011 Accepted: 25 January 2012 Published: 25 January 2012

\section{References}

1. Kohler A, Hurt E: Exporting RNA from the nucleus to the cytoplasm. Nat Rev Mol Cell Biol 2007, 8:761-773.

2. Moore MJ, Proudfoot NJ: Pre-mRNA processing reaches back to transcription and ahead to translation. Cell 2009, 136:688-700. 
3. Zhong XY, Wang P, Han J, Rosenfeld MG, Fu XD: SR proteins in vertical integration of gene expression from transcription to RNA processing to translation. Mol Cell 2009, 35:1-10.

4. Kress TL, Yoon YJ, Mowry KL: Nuclear RNP complex assembly initiates cytoplasmic RNA localization. J Cell Biol 2004, 165:203-211.

5. Stoltzfus CM: Synthesis and processing of avian sarcoma retrovirus RNA Adv Virus Res 1988, 35:1-38.

6. Arrigo S, Beemon K: Regulation of Rous sarcoma virus RNA splicing and stability. Mol Cell Biol 1988, 8:4858-4867.

7. Katz RA, Kotler M, Skalka AM: cis-acting intron mutations that affect the efficiency of avian retroviral RNA splicing: implication for mechanisms of control. J Virol 1988, 62:2686-2695.

8. Katz RA, Skalka AM: Control of retroviral RNA splicing through maintenance of suboptimal processing signals. Mol Cell Biol 1990, 10:696-704.

9. Gruter P, Tabernero C, von Kobbe C, Schmitt C, Saavedra C, Bachi A Wilm M, Felber BK, Izaurralde E: TAP, the human homolog of Mex67p, mediates CTE-dependent RNA export from the nucleus. Mol Cell 1998, 1:649-659.

10. Hadzopoulou-Cladaras M, Felber BK, Cladaras C, Athanassopoulos A, Tse A, Pavlakis GN: The rev (trs/art) protein of human immunodeficiency virus type 1 affects viral mRNA and protein expression via a cis-acting sequence in the env region. J Virol 1989, 63:1265-1274

11. Malim MH, Hauber J, Le SY, Maizel JV, Cullen BR: The HIV-1 rev transactivator acts through a structured target sequence to activate nuclear export of unspliced viral mRNA. Nature 1989, 338:254-257.

12. Neville M, Stutz F, Lee L, Davis LI, Rosbash M: The importin-beta family member $\mathrm{Crm} 1 \mathrm{p}$ bridges the interaction between Rev and the nuclear pore complex during nuclear export. Curr Biol 1997, 7:767-775.

13. Mertz JA, Simper MS, Lozano MM, Payne SM, Dudley JP: Mouse mammary tumor virus encodes a self-regulatory RNA export protein and is a complex retrovirus. J Virol 2005, 79:14737-14747.

14. Mertz JA, Chadee AB, Byun H, Russell R, Dudley JP: Mapping of the functional boundaries and secondary structure of the mouse mammary tumor virus Rem-responsive element. J Biol Chem 2009, 284:25642-25652.

15. Hofacre A, Nitta T, Fan H: Jaagsiekte sheep retrovirus encodes a regulatory factor, Rej, required for synthesis of Gag protein. J Virol 2009, 83:12483-12498.

16. Cardiff RD, Kenney N: Mouse mammary tumor biology: a short history. Adv Cancer Res 2007, 98:53-116.

17. Kinyamu HK, Archer TK: Modifying chromatin to permit steroid hormone receptor-dependent transcription. Biochim Biophys Acta 2004, 1677:30-45.

18. Indik S, Gunzburg WH, Salmons B, Rouault F: A novel, mouse mammary tumor virus encoded protein with Rev-like properties. Virology 2005, 337:1-6

19. Mullner M, Salmons B, Gunzburg WH, Indik S: Identification of the Remresponsive element of mouse mammary tumor virus. Nucleic Acids Res 2008, 36:6284-6294.

20. D'Agostino DM, Felber BK, Harrison JE, Pavlakis GN: The Rev protein of human immunodeficiency virus type 1 promotes polysomal association and translation of gag/pol and vpu/env mRNAs. Mol Cell Biol 1992, 12:1375-1386.

21. Schwartz S, Felber BK, Pavlakis GN: Distinct RNA sequences in the gag region of human immunodeficiency virus type 1 decrease RNA stability and inhibit expression in the absence of Rev protein. J Virol 1992, 66:150-159.

22. Perales C, Carrasco L, Gonzalez ME: Regulation of HIV-1 env mRNA translation by Rev protein. Biochim Biophys Acta 2005, 1743:169-175.

23. Schneider R, Campbell M, Nasioulas G, Felber BK, Pavlakis GN: Inactivation of the human immunodeficiency virus type 1 inhibitory elements allows Rev-independent expression of Gag and Gag/protease and particle formation. J Virol 1997, 71:4892-4903.

24. Butsch M, Hull S, Wang Y, Roberts TM, Boris-Lawrie K: The 5' RNA terminus of spleen necrosis virus contains a novel posttranscriptional control element that facilitates human immunodeficiency virus Rev/RREindependent Gag production. J Virol 1999, 73:4847-4855

25. Hull S, Boris-Lawrie K: RU5 of Mason-Pfizer monkey virus $5^{\prime}$ long terminal repeat enhances cytoplasmic expression of human immunodeficiency virus type 1 gag-pol and nonviral reporter RNA. J Virol 2002, 76:10211-10218.
26. Hartman TR, Qian S, Bolinger C, Fernandez S, Schoenberg DR, BorisLawrie K: RNA helicase $A$ is necessary for translation of selected messenger RNAs. Nat Struct Mol Biol 2006, 13:509-516.

27. Bolinger C, Sharma A, Singh D, Yu L, Boris-Lawrie K: RNA helicase A modulates translation of HIV-1 and infectivity of progeny virions. Nucleic Acids Res 38:1686-1696

28. Shackleford GM, Varmus HE: Construction of a clonable, infectious, and tumorigenic mouse mammary tumor virus provirus and a derivative genetic vector. Proc Natl Acad Sci USA 1988, 85:9655-9659.

29. Salmons B, Groner B, Calberg-Bacq CM, Ponta H: Production of mouse mammary tumor virus upon transfection of a recombinant proviral DNA into cultured cells. Virology 1985, 144:101-114.

30. Brookes S, Placzek M, Moore R, Dixon M, Dickson C, Peters G: Insertion elements and transitions in cloned mouse mammary tumour virus DNA: further delineation of the poison sequences. Nucleic Acids Res 1986, 14:8231-8245.

31. Zabransky A, Sakalian M, Pichova I: Localization of self-interacting domains within betaretrovirus Gag polyproteins. Virology 2005, 332:659-666

32. Grote A, Hiller K, Scheer M, Munch R, Nortemann B, Hempel DC, Jahn D: JCat: a novel tool to adapt codon usage of a target gene to its potential expression host. Nucleic Acids Res 2005, 33:W526-531.

33. Swanson CM, Puffer BA, Ahmad KM, Doms RW, Malim MH: Retroviral mRNA nuclear export elements regulate protein function and virion assembly. EMBO J 2004, 23:2632-2640.

34. Bhadra S, Lozano MM, Payne SM, Dudley JP: Endogenous MMTV proviruses induce susceptibility to both viral and bacterial pathogens. PLoS Pathog 2006, 2:e128.

35. Indik S, Gunzburg WH, Salmons B, Rouault F: Mouse mammary tumor virus infects human cells. Cancer Res 2005, 65:6651-6659.

36. Okeoma CM, Low A, Bailis W, Fan HY, Peterlin BM, RosS SR: Induction of APOBEC3 in vivo causes increased restriction of retrovirus infection. $J$ Virol 2009, 83:3486-3495.

37. Vaidya $A B$, Taraschi NE, Tancin SL, Long CA: Regulation of endogenous murine mammary tumor virus expression in C57BL mouse lactating mammary glands: transcription of functional mRNA with a block at the translational level. J Virol 1983, 46:818-828.

38. Klein KC, Reed JC, Lingappa JR: Intracellular destinies: degradation, targeting, assembly, and endocytosis of HIV Gag. AIDS Rev 2007, 9:150-161

39. Schwartz S, Campbell M, Nasioulas G, Harrison J, Felber BK, Pavlakis GN: Mutational inactivation of an inhibitory sequence in human immunodeficiency virus type 1 results in Rev-independent gag expression. J Virol 1992, 66:7176-7182.

40. Groom HC, Anderson EC, Lever AM: Rev: beyond nuclear export. J Gen Virol 2009, 90:1303-1318.

41. Deroo BJ, Archer TK: Glucocorticoid receptor-mediated chromatin remodeling in vivo. Oncogene 2001, 20:3039-3046.

42. Bolinger C, Boris-Lawrie K: Mechanisms employed by retroviruses to exploit host factors for translational control of a complicated proteome. Retrovirology 2009, 6:8.

43. Simpson SB, Zhang L, Craven RC, Stoltzfus CM: Rous sarcoma virus direct repeat cis elements exert effects at several points in the virus life cycle. J Virol 1997, 71:9150-9156.

44. Simpson SB, Guo W, Winistorfer SC, Craven RC, Stoltzfus CM: The upstream, direct repeat sequence of Prague A Rous sarcoma virus is deficient in mediating efficient Gag assembly and particle release. Virology 1998, 247:86-96.

45. Ogert RA, Lee $L H$, Beemon KL: Avian retroviral RNA element promotes unspliced RNA accumulation in the cytoplasm. J Virol 1996, 70:3834-3843.

46. Berberich SL, Macias M, Zhang L, Turek LP, Stoltzfus CM: Comparison of Rous sarcoma virus RNA processing in chicken and mouse fibroblasts: evidence for double-spliced RNA in nonpermissive mouse cells. J Virol 1990, 64:4313-4320.

47. Vogt VM, Bruckenstein DA, Bell AP: Avian sarcoma virus gag precursor polypeptide is not processed in mammalian cells. J Virol 1982, 44:725-730.

48. Nasioulas G, Hughes SH, Felber BK, Whitcomb JM: Production of avian leukosis virus particles in mammalian cells can be mediated by the interaction of the human immunodeficiency virus protein Rev and the Rev-responsive element. Proc Natl Acad Sci USA 1995, 92:11940-11944. 
49. Swanson CM, Sherer NM, Malim MH: SRp40 and SRp55 promote the translation of unspliced human immunodeficiency virus type 1 RNA. J Virol 84:6748-6759.

50. Pollard WW, Malim MH: The HIV-1 Rev protein. Annu Rev Microbiol 1998, 52:491-532

51. Zolotukhin AS, Michalowski D, Bear J, Smulevitch SV, Traish AM, Peng R, Patton J, Shatsky IN, Felber BK: PSF acts through the human immunodeficiency virus type $1 \mathrm{mRNA}$ instability elements to regulate virus expression. Mol Cell Biol 2003, 23:6618-6630.

52. Mertz JA, Lozano MM, Dudley JP: Rev and Rex proteins of human complex retroviruses function with the MMTV Rem-responsive element. Retrovirology 2009, 6:10.

53. Lu XB, Heimer J, Rekosh D, Hammarskjold ML: U1 small nuclear RNA plays a direct role in the formation of a rev-regulated human immunodeficiency virus env mRNA that remains unspliced. Proc Natl Acad Sci USA 1990, 87:7598-7602.

54. Cochrane AW, McNally MT, Mouland AJ: The retrovirus RNA trafficking granule: from birth to maturity. Retrovirology 2006, 3:18.

55. Swanson CM, Malim MH: Retrovirus RNA trafficking: from chromatin to invasive genomes. Traffic 2006, 7:1440-1450

56. Suhasini M, Reddy TR: Cellular proteins and HIV-1 Rev function. Curr HIV Res 2009, 7:91-100.

57. Zabransky A, Hadravova R, Stokrova J, Sakalian M, Pichova I: Premature processing of mouse mammary tumor virus Gag polyprotein impairs intracellular capsid assembly. Virology 2009, 384:33-37.

58. Fasel N, Buetti E, Firzlaff J, Pearson K, Diggelmann H: Nucleotide sequence of the $5^{\prime}$ noncoding region and part of the gag gene of mouse mammary tumor virus; identification of the $5^{\prime}$ splicing site for subgenomic mRNAs. Nucleic Acids Res 1983, 11:6943-6955.

59. Fasel N, Pearson K, Buetti E, Diggelmann H: The region of mouse mammary tumor virus DNA containing the long terminal repeat includes a long coding sequence and signals for hormonally regulated transcription. EMBO J 1982, 1:3-7.

60. Redmond SM, Dickson C: Sequence and expression of the mouse mammary tumour virus env gene. EMBO J 1983, 2:125-131.

61. Xu L, Sakalian M, Shen Z, Loss G, Neuberger J, Mason A: Cloning the human betaretrovirus proviral genome from patients with primary biliary cirrhosis. Hepatology 2004, 39:151-156.

62. Sakalian M, Rapp ND: Rescue of internal scaffold-deleted Mason-Pfizer monkey virus particle production by plasma membrane targeting. Virology 2006, 345:317-327.

doi:10.1186/1742-4690-9-8

Cite this article as: Boeras et al.: Translation of MMTV Gag requires nuclear events involving splicing motifs in addition to the viral Rem protein and RmRE. Retrovirology 2012 9:8.

\section{Submit your next manuscript to BioMed Central and take full advantage of:}

- Convenient online submission

- Thorough peer review

- No space constraints or color figure charges

- Immediate publication on acceptance

- Inclusion in PubMed, CAS, Scopus and Google Scholar

- Research which is freely available for redistribution

Submit your manuscript at www.biomedcentral.com/submit
Biomed Central 